\title{
Influence of GalnP ordering on the electronic quality of concentrator solar cells
}

\author{
I. Garcia , I. Rey-Stolle , C. Algora , W. Stolz , K. Volz \\ ${ }^{a}$ Institute) de Energia Solar, E.T.S.I. Telecomunicacion, Universidad Politecnica de Madrid, Spain \\ ${ }^{b}$ Material Sciences Center-Central Technology Laboratory and Department of Physics, Philipps University Marburg, 35032 Marburg, Germany
}

Keywords:

Al. Ordering

A3. Metalorganic vapour phase epitaxy

B1 CalnP

\begin{abstract}
A B S T R A C T
The ordering phenomenon produces a reduction in the band gap of the GalnP material. Though a drawback for many optoelectronic applications, ordering can be used as an additional degree of material and device engineering freedom. The performance of the record efficiency GalnP/GaAs/Ge multijunction solar cells depends on the quality and design of the GalnP top cell, which can be affected also by ordering. The tradeoff existing between band gap and minority carrier properties, and the possibility of creating a back surface field (BSF) structure based on an order-disorder GalnP heterostructure makes the study of the ordering appealing for solar cell applications. In this work, the ordering dependency with the growth conditions and substrate orientation is studied. The results obtained are presented to enrich and extend the data available in the literature. Then the properties of order-disorder GalnP heterostructures are assessed by using them as BSF in GalnP concentrator solar cells. The external

quantum efficiency (EQE) shows a good behavior of these BSF layers, but unexpectedly poor electronic quality in the active layers. Although the exact origin of this problem remains to be known, it is attributed to traps introduced by the ordered/disordered domains matrix or growth native defects. $\mathrm{BQE}$ measurements with bias light show a recovery of the minority carrier properties, presumably due to the saturation of the traps.
\end{abstract}

\section{Introduction}

GalnP grown lattice matched to GaAs exhibits anomalous changes in the band gap depending on the growth conditions and the substrate misorientation. These changes are the result of the spontaneous ordering during the growth of the cation-site elements ( $\mathrm{Ga}$ and $\mathrm{In}$ ) in planes parallel to the (111). This gives rise to a $\mathrm{Cu}-\mathrm{Pt}$ structure, which results in a lowering of the band gap of the material, whose exact value depends on the degree of ordering. Reductions above $100 \mathrm{meV}$ can appear. This is sometimes a drawback due to the red-shift of the emission of optoelectronic devices made with ordered GalnP. However, the relation between ordering and band gap provides a new degree of freedom when working with GalnP. For example, sharp O/D GalnP structures can be created by just changing the tertiarybutylphosphine (TBP) partial pressure during growth

Three-junction GalnP/GaAs/Ge concentrator solar cells have achieved record efficiencies exceeding $40 \%$. The theoretical calculations for this combination of materials indicate that, to achieve maximum efficiencies, the band gap of the GalnP top cell should be as high as possible, i.e., the GalnP should be completely disordered . However, the MOVPE growth conditions that produce such a material have deleterious effects on other important aspects like surface morphology or minority carrier properties, which determine the performance of the solar cell. In practice, the usual band gap of the GalnP lattice matched to GaAs suitable for solar cell applications is $1.85 \mathrm{eV}$. On the other hand, a current matched multijunction device must be obtained to maximize its conversion efficiency. Since at the same time, it is desirable to have a shortcircuit current as high as possible, the GalnP top cell must absorb the minimum amount of light with maximum conversion efficiency. The usual technique to achieve this consists on thinning the top cell base layer. This makes this layer more sensitive to the quality of its interface with the layer underneath. To improve the conversion efficiency, an effective back surface field (BSF) layer must be inserted. Again, GalnP ordering can play a role in this aspect, for it is possible to grow a BSF structure based on an order-disorder GalnP layer stack. To achieve this, the growth conditions must be chosen carefully for the active (emitter and base) and BSF layers. The description and results of such a task is the aim of next sections.

\section{Experimental procedure}

All samples were grown in a horizontal, low pressure, AIX200 reactor. The source materials were trimethylgallium (TMGa), 
trimethylindium (TMIn), tertiarybutylphospine (TBP), tertiarybutylarsine (TBAs), trimethylaluminium (TMAl), diethylzinc (DEZn) and ditertiarybutylsilane (DTBSi $\left.{ }_{2}\right)$. Using TBP is especially advantageous in this case, because it allows the achievement of sharp order-disorder GaInP heterostructures with a variation of the $\mathrm{V} / \mathrm{III}$ ratio during growth, which is not possible if $\mathrm{PH}_{3}$ is used [2]. The work pressure was $50 \mathrm{mBar}$ and the temperature was corrected using the $\mathrm{Al} / \mathrm{Si}$ eutectic method. All the experiments were preceded by a cleaning and coating run consisting on a baking of the susceptor at $800^{\circ} \mathrm{C}$ and a deposition of about $1 \mu \mathrm{m}$ of GaAs. This was done to prevent the In and P coming from the GaInP terminated runs to affect the following experiments. After the epitaxial process, all the samples were systematically characterized with the high-resolution X-ray diffraction (HRXRD), room-temperature photoluminescence (RT-PL) and AFM techniques. For the solar cell external quantum efficiency (EQE) measurements, a monochromator-based system is employed with a chopped light beam. The detection is made by means of the lockin technique.

\section{Ordering vs. growth conditions and substrate misorientation}

It is known that ordering in GaInP grown by metal organic chemical vapour deposition (MOCVD) depends on the growth conditions and substrate misorientation. Some general trends have been found for the relation between a growth parameter and the ordering achieved [4]. However, the interrelation between the growth parameters and the ordering is intricate, and a tendency, observed for a given set of growth parameters can change when modifying any of them.

In this work, a number of experiments are performed in order to determine the ordering level in GaInP for a set of growth conditions and GaAs substrates. The semiconductor structures used for the study consist of $300 \mathrm{~nm}$ thick undoped GaInP layers lattice matched to GaAs grown on top of $250 \mathrm{~nm}$ thick buffer layers. Growth temperatures from 580 to $650{ }^{\circ} \mathrm{C}$ and V/III ratios from 2 to over 100 are explored, for three different substrate misorientations. The growth rate was kept constant at $1.5 \mu \mathrm{m} / \mathrm{h}$. The cooling down of the sample was done under a TBP stabilized atmosphere. The assessment of the ordering is made in terms of the absolute difference between the band gap of the GaInP test layers at room temperature, and the band gap of a completely disordered GaInP material, whose value at $295 \mathrm{~K}$ was taken to be $1.91 \mathrm{eV}$ for the GaInP lattice matched to GaAs [6]. The (optical) band gap of the test layers was estimated using RT-PL. The value obtained was then corrected for the observed small composition deviations from the lattice matched to GaAs one, measured by HRXRD [7]. The surface morphology is characterized using AFM. A summary of the experiments is presented in Figs. 1 and 2. Some relevant conclusions for our purposes drawn from the data can be summarized as follows:

- For all temperatures and substrates, the ordering increases when the $\mathrm{V} / \mathrm{III}$ ratio is increased, more pronouncedly for values below 20.

- The maximum ordering is achieved with $6^{\circ} \rightarrow(111) \mathrm{B}$ substrates at $650^{\circ} \mathrm{C}$ and V/III $>100$.

- The maximum variation of the ordering changing only the $\mathrm{V} / \mathrm{III}$ ratio $(50 \mathrm{meV})$ is achieved with $6^{\circ} \rightarrow(111) \mathrm{B}$ substrates at $625^{\circ} \mathrm{C}$.

- The roughness increases as temperature increases, for all the substrates.

- The dependency of the roughness with the V/III ratio is minimum with the $6^{\circ} \rightarrow(111) \mathrm{B}$ substrates.

- For V/III ratios from about 20 to higher values, the minimum roughness is achieved with $6^{\circ} \rightarrow(111) \mathrm{B}$ substrates.

It is noteworthy the data scatter present in the study of the (111)B substrates. We believe that spatial inhomogeneities are not responsible for the scatter since the PL maps performed on the samples resulted very uniform. On the other hand, we think this effect is the result of a high sensibility of ordering to the growth temperature for the set of growth conditions used in the experiments. The variation of the growth temperature is
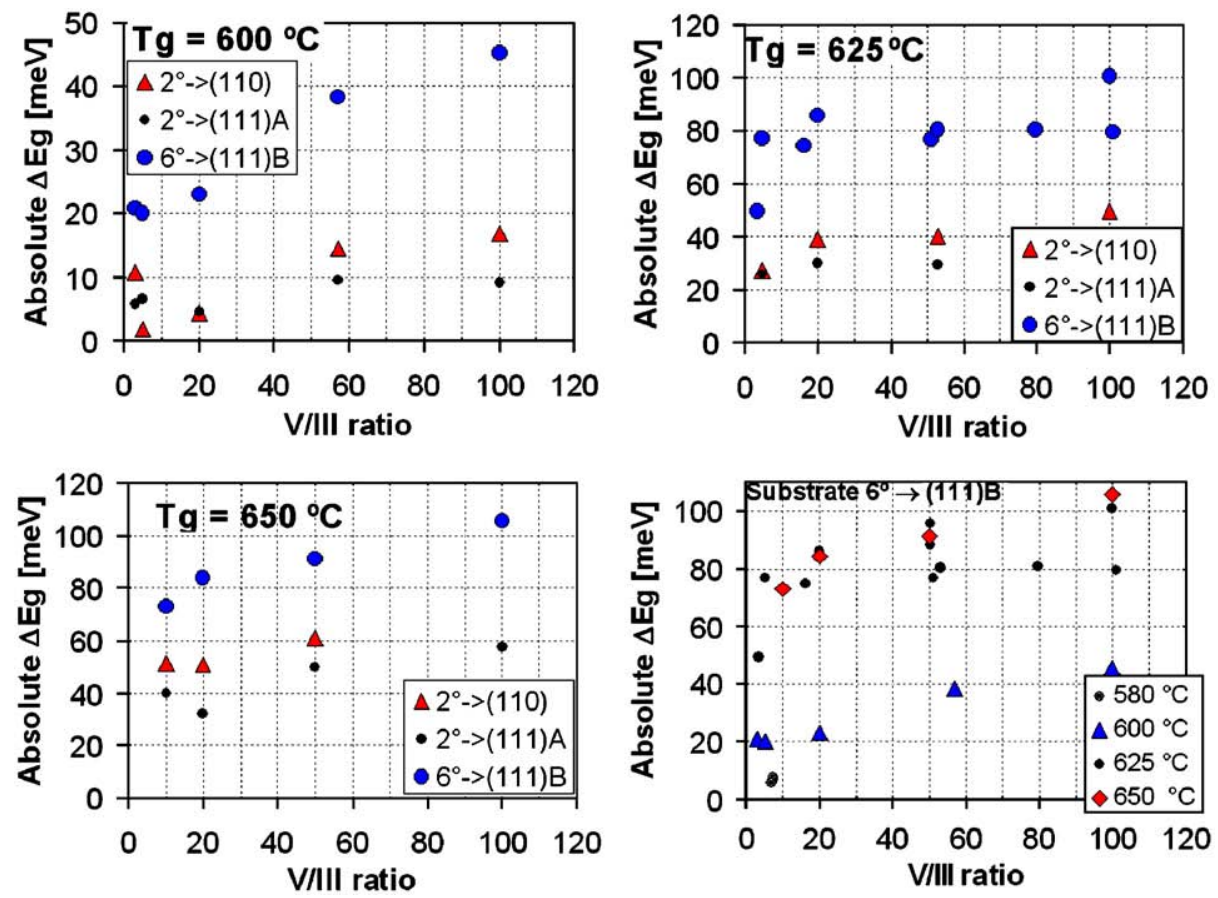

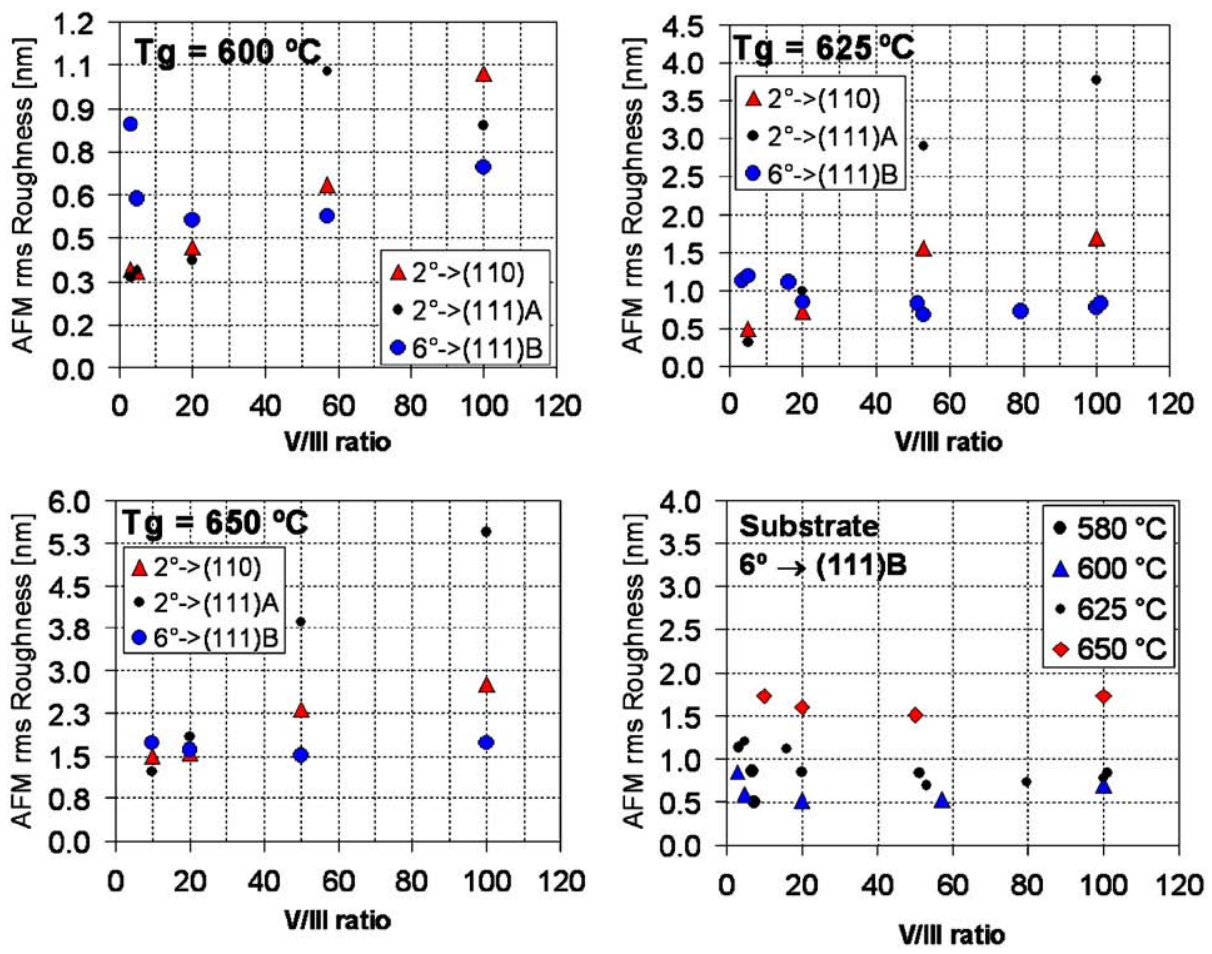

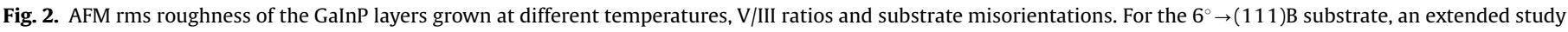
was carried out

presumably coming from the fact that wafer pieces of different sizes were used for each experiment and they were positioned in a slightly different part of the $2^{\prime \prime}$ recess of the satellite during growth.

The aim of the GaInP layers grown at $580^{\circ} \mathrm{C}$ and low V/III ratio was to achieve the most completely disordered layer possible. It is observed that the absolute band-gap energy difference of such a layer is about $7 \mathrm{meV}$. An attempt to further decrease this value by decreasing the V/III ratio gave rise to a GaInP layer of such a bad morphology that it appeared milky to the naked eye. Thus, the layers grown at $580{ }^{\circ} \mathrm{C}$ and a V/III ratio of about 7 were considered as the less ordered layers achievable in the range of growth conditions explored.

\section{GaInP concentrator solar cells grown with ordered GaInP and a BSF based on an order-disorder heterostructure}

After the results obtained in the previous experiments, the substrates oriented $6^{\circ} \rightarrow(111) \mathrm{B}$ were considered more appropriate for the growth of solar cells based on ordered GaInP for the active layers and on an ordered-disordered heterostructure used as the BSF. On the one hand, the ability of achieving a highly ordered layer and the widest band gap difference is advantageous for the growth of the BSF layer based on an ordered-disordered heterostructure. On the other hand, the surface morphology studies suggest that $6^{\circ} \rightarrow(111) \mathrm{B}$ oriented substrates are the most flexible in terms of roughness at different growth temperatures and $\mathrm{V} / \mathrm{III}$ ratios.

The structure of the solar cells grown is shown in Fig. 3. The values chosen for the active layers thicknesses and doping levels are the standard ones that produce high efficiencies in multijunction solar cells $[3,5]$. As for the growth conditions they were chosen keeping in mind the tradeoff imposed by the ordering necessary in the active and BSF layers to be able to produce an sufficient conduction-band discontinuity to serve as an effective

\begin{tabular}{|c|}
\hline CAP GaAs:Si \\
\hline Window AllnP:Si $20 \mathrm{~nm} \mathrm{~N} \geq 5 e 17$ \\
\hline Base GalnP:Zn $600 \mathrm{~nm} \mathrm{~N}_{\mathrm{A}}=1 \mathrm{e} 17$ \\
\hline BSF GalnP:Zn $75 \mathrm{~nm} \mathrm{~N}_{\mathrm{A}}=3 \mathrm{e} 18$ \\
\hline GaAs: $Z \mathrm{Zn}$ substrate \\
\hline
\end{tabular}

reflector for minority carriers (holes), and the quality of the photoactive layers, i.e., the emitter and base. Three structures were grown and a summary of their growth conditions can be found in Table 1 . The conditions chosen for the active layers also appear to be the optimum to achieve a good electronic quality, since the surface roughness is minimum and the RT-PL peak intensity was in the range of the highest values (not shown here for brevity).

The solar cell structures were then processed into concentrator solar cell devices with $1 \mathrm{~mm}^{2}$ active area and an inverted square front grid. The quality of the semiconductor structure was assessed by way of the analysis of the EQE of the device. The curves obtained are shown in Fig. 4. The EQE of another GaInP solar cell with similar thicknesses and doping levels but grown with $\mathrm{PH}_{3}$ as group-V precursor and at different temperatures and $\mathrm{V} / \mathrm{III}$ ratios which produce less ordering in the GaInP is also 
Table 1

Growth details of the GaInP solar cells grown

\begin{tabular}{|c|c|c|c|c|c|c|c|}
\hline \multirow[t]{2}{*}{ Run } & \multicolumn{2}{|c|}{$\begin{array}{l}\text { Photoactive layers growth } \\
\text { conditions }\end{array}$} & \multirow[t]{2}{*}{$\begin{array}{l}\text { Photoactive layers } \\
\text { nominal } E_{\mathrm{g}}(\mathrm{eV})\end{array}$} & \multicolumn{2}{|c|}{ BSF layers growth conditions } & \multirow[t]{2}{*}{$\begin{array}{l}\text { BSF layer nominal } \\
E_{\mathrm{g}}(\mathrm{eV})\end{array}$} & \multirow[t]{2}{*}{$\begin{array}{l}\text { Base-BSF } E_{\mathrm{g}} \\
\text { difference (meV) }\end{array}$} \\
\hline & $T_{\mathrm{g}}\left({ }^{\circ} \mathrm{C}\right)$ & V/III & & $T_{\mathrm{g}}\left({ }^{\circ} \mathrm{C}\right)$ & V/III & & \\
\hline$\# 1$ & & & & 625 & 3 & 1.860 & 35 \\
\hline$\sharp 2$ & 625 & 50 & 1.825 & 580 & 7 & 1.903 & 78 \\
\hline$\# 3$ & & & & 625 & 50 ( $\uparrow$ doping $)$ & 1.825 & 0 \\
\hline
\end{tabular}

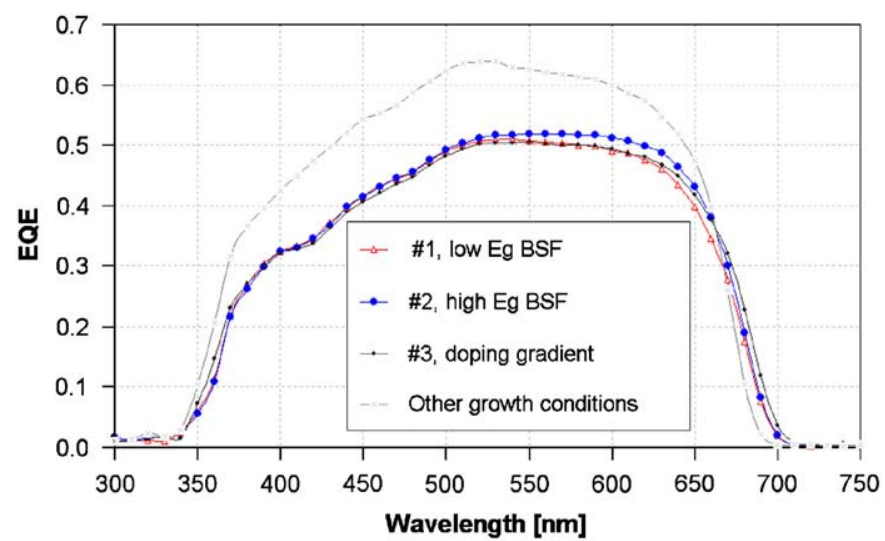

Fig. 4. External quantum efficiency of the GaInP solar cells fabricated. The EQE of a similar solar cell with less ordered GaInP and grown with $\mathrm{PH}_{3}$ is shown for comparison.

plotted for comparison purposes. Since the GaInP compositions obtained with HRXRD showed to be quite close, the difference in ordering level is deduced directly from the absorption cut-off wavelength in the EQE, which is higher in the case of more ordered material. About the BSF quality, the differences between the three structures grown are clearly noticeable. The structure with the higher conduction-band discontinuity produced by the highly disordered BSF layer ( $\$ 2$ ) shows the best response in the lowest energy region, which can be ascribed to a more effective reflection of the minority carriers. The behavior of the BSF layer generated with a change in the V/III ratio from the BSF to the base layer is not better than that of the BSF created with a doping level gradient. This is consistent with the data presented in Table 1 . The difference of just $35 \mathrm{meV}$ between the band gap of the BSF and base layers, clearly below the minimum $2 \mathrm{kT}$ value necessary to serve as an effective minority carrier barrier, explains the worse performance of this BSF layer.

The low amplitude of the EQE of the solar cells grown in this study is remarkable. This affects directly the short-circuit current of the solar cell. The worse response in the solar cells grown with more ordered GaInP is believed to be due to poor minority carrier properties of this material. In fact, the simulations carried out using the Hovel model [8] give rise to very close fits of these curves if very low minority carrier lifetimes or mobilities (i.e. low diffusion lengths) are used in the calculations. The origin of these poor minority carrier properties cannot be blamed on structural defects coming from an excessive lattice mismatch, since the HRXRD measurements showed a $\Delta a / a$ value below $7.5 \times 10^{-5}$. The use of TBP, which in the past was found to be problematic in obtaining phosphide materials with high minority carrier properties [9], is nowadays improved to an extent that makes it appropriate for growing high-quality material [10]. Moreover, oxygen, carbon and atomic hydrogen SIMS profiling performed on the samples grown in this study (see Fig. 5) show a residual
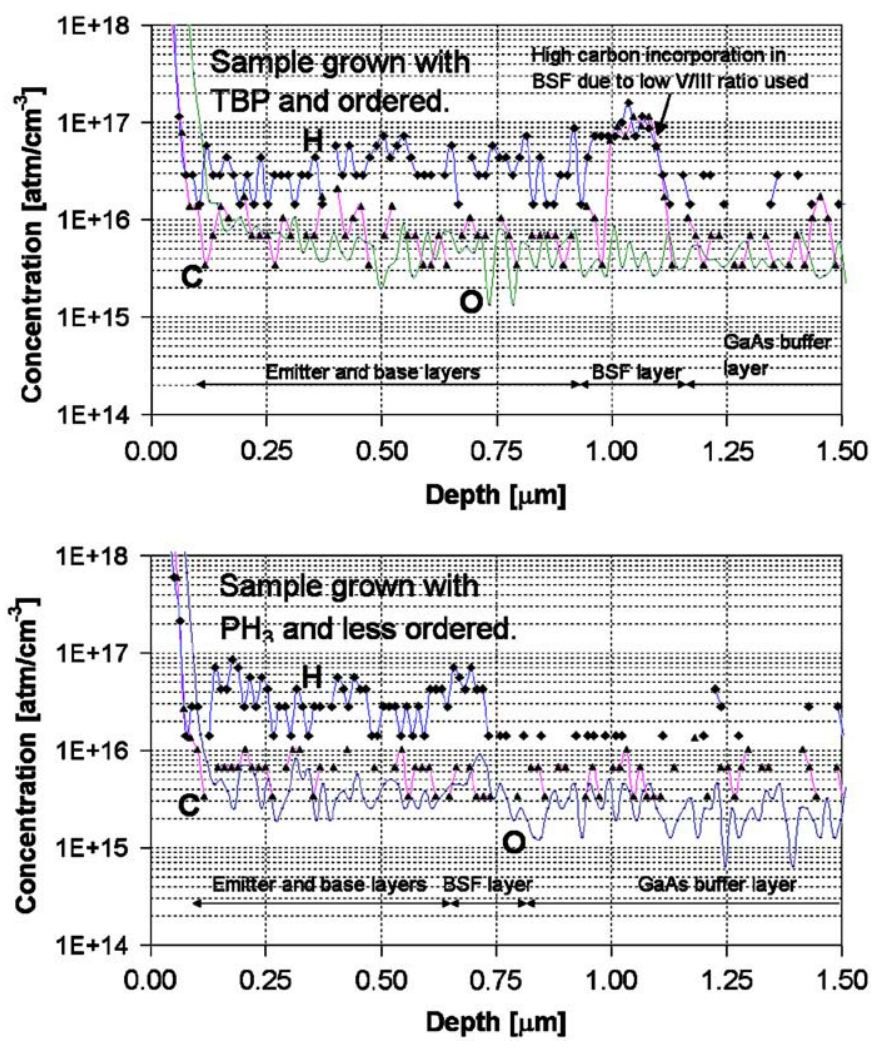

Fig. 5. Oxygen, carbon and atomic hydrogen SIMS profile performed on the GaInP solar cells grown using TBP and $\mathrm{PH}_{3}$.

concentration of all these elements very similar in the solar cells grown with $\mathrm{PH}_{3}$ and TBP, and with quantities below any harmful value. Thus, the use of TBP itself does not appear to be the origin of the poor minority carrier properties found. On the other hand, the ordering in GaInP is known to produce a reduction in the electronic quality of the GaInP layers due to the formation of traps arising from the order/disorder domain matrix [11]. However, other sources of the poor electronic quality like native defects due to the growth conditions chosen cannot be discarded. A deeper study of the causes falls out of the scope and length of this paper, but it is currently being studied.

The solar cells fabricated are aimed to work under concentrated light. However, the standard EQE measurements are carried out at low- light densities, much below the 1 sun equivalent (in our case it is as low as $1 / 20$ suns). In some cases, it has been found that the EQE response improves if a light bias is applied to the solar cell during the measurement [12] due to the saturation of the recombination centers that worsen the response of the solar cell. To study this effect on the solar cells we are analyzing in this work, a $635 \mathrm{~nm}$ laser light bias equivalent to the irradiance of 7 suns was applied to the solar cells during the EQE measurement. 


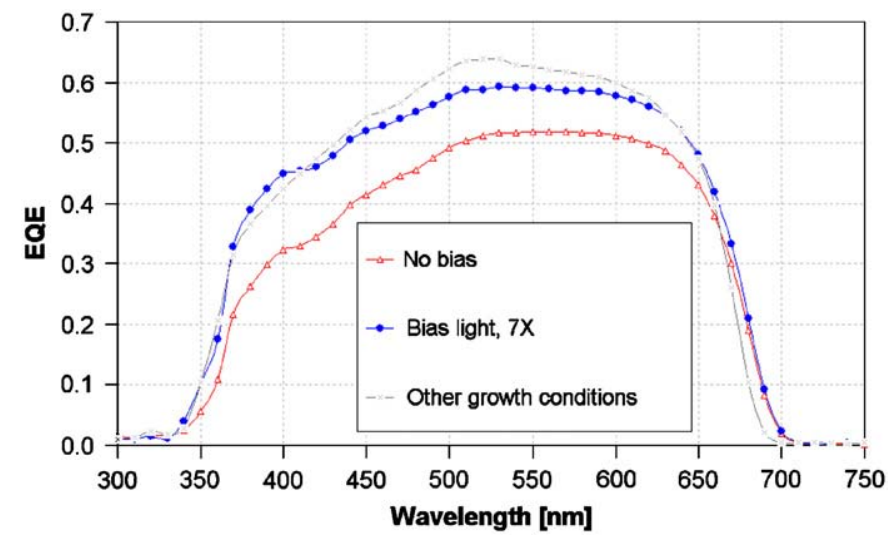

The result for one of the samples is shown in Fig. 6. For the other samples, the result is similar. The important improvement in the EQE can be seen, which supports the idea of a trap related poor EQE performance. In the improved EQE curve, a higher improvement in the response for high photon energies can also be appreciated. This is due to the laser light wavelength used, with which the excitation is higher in the upper layers. Although it is necessary to study more in depth the characteristics of the traps involved, it appears that they may not be necessarily a problem for the solar cell performance when working under concentrated light.

\section{Summary and conclusions}

In this work, the relation between growth conditions, ordering and surface morphology for different substrate misorientations have been studied. For the V/III ratio and temperature ranges explored, it has been found that the $6^{\circ} \rightarrow(111) \mathrm{B}$ substrates allow the maximum variation or ordering changing only the $\mathrm{V} / \mathrm{III}$ ratio. These susbstrates also show the minimum surface-roughness sensitivity to the growth conditions. The minimum ordering with this substrate was obtained at a growth temperature of $580^{\circ} \mathrm{C}$ and a V/III ratio of 7. This highly disordered layer was used as a BSF in a GaInP concentrator solar cell. The EQE result show an improved performance of this BSF with respect to other ones based on a doping gradient or a more ordered GaInP layer. The electronic quality of the active layer in the solar cells fabricated was found to be poor, compared to other solar cells grown with $\mathrm{PH}_{3}$ and a less ordered GaInP. The SIMS measurements performed appear not to point to TBP as the origin of this problem. On the other hand, the traps causing the bad electronic quality are thought to come from growth native defects or the order/disordered domains matrix, although the exact origin remains to be determined. From the concentration EQE measurements performed, it was found that the electronic properties were improved with the light bias applied. The trap saturation is thought to be the origin of this behavior. Thus, it can be concluded that the traps introduced by the ordering in the GaInP are not necessarily a problem for the conversion efficiency when the solar cells are aimed to work at high concentrations. The particular characteristics of these traps are currently being studied.

\section{Acknowledgements}

This paper has been supported by the European Commission under contract SES6-CT-2003-502620 (FULLSPECTRUM project). The Spanish Ministerio de Educación y Ciencia has also contributed with the CONSOLIDER-INGENIO 2010 program by means of the GENESIS FV project and also with the research projects with references TEC2005-02745, TEC2004-22300-E, and TEC200729630-E as well as the Comunidad de Madrid under NUMANCIA program (S-505/ENE/0310).

\section{References}

[1] A. Gomyo, K. Kobayashi, S. Kawata, I. Hino, T. Suzuki, T. Yuasa, J. Crystal Growth 77 (1986) 367.

[2] Y.S. Chun, H. Murata, I.H. Ho, T.C. Hsu, G.B. Stringfellow, J. Crystal Growth 170 (1997) 263.

[3] R.R. King, et al., in: Proceedings of the Fourth International Conference on Solar Concentrators, 2007, p. 8.

[4] J.M. Olson, et al., in: A. Luque, S. Hegedus (Eds.), Handbook of Photovoltaic Science and Engineering, Wiley, 2003 (Chapter 9).

[5] K.A. Bertness, A. Sarah, R. Kurtz, D.J. Friedman, A.E. Kibbler, C. Kramer, J.M. Olson, Appl. Phys. Lett. 65 (1995) 989.

[6] M.C. Delong, et al., Appl. Phys. Lett. 66 (1995) 3158.

[7] C.P. Kuo, S.K. Vong, R.M. Cohen, G.B. Stringfellow, J. Appl. Phys. 57 (1985) 5428.

[8] H.J. Hovel, J.M. Woodall, in: Proceedings of the 10th IEEE PVSC (1973) p. 25.

[9] R.W. Hoffman, N.S. Fatemi, D.M. Wilt, P.P. Jenkins, D. Brinker, D.A. Scheiman, in: Proceedings of the 24th IEEE PV Specialists Conference IEEE, Waikoloa, HI, USA, 1994, p. 1882

[10] H.-J. Schimper, Z. Kollonitsch, K. Möller, U. Seidel, U. Bloeck, K. Schwarzburg, F. Willig, T. Hannappel, J. Crystal Growth 287 (2006) 642

[11] S.F. Yoon, K.W. Mah, H.Q. Zheng, P.H. Zhang, J. Crystal Growth 208 (2000) 197.

[12] I. Garcia, I. Rey-Stolle, B. Galiana, C. Algora, in: Proceedings of the Fourth World Conference on Photovoltaic Energy Conversion (2006) p. 830. 